\title{
Efficacy and Outcomes of Intraoperative Extensive Lavage Plus Surgery Versus Surgery Alone in Patients with Advanced Gastric Cancer: A Multicenter Randomized Controlled Trial
}

\section{Li-xiang Zhang}

First Affiliated Hospital of Anhui Medical University

Chuan-hong Li

First Affiliated Hospital of Anhui Medical University

Jun Ma

Anqing Munipital Hospital

Lei Chen

Wenzhou Medical College First Affiliated Hospital: The First Affiliated Hospital of Wenzhou Medical University

\section{Zhi-jian Wei}

First Affiliated Hospital of Anhui Medical University

\section{A-Man Xu}

First Affiliated Hospital of Anhui Medical University

Wenxiu Han ( $\nabla$ hwxhbh@126.com )

First Affiliated Hospital of Anhui Medical University https://orcid.org/0000-0002-5335-9645

\section{Research}

Keywords: EIPL, advanced gastric cancer, prognosis, recurrence, OS

Posted Date: July 19th, 2021

DOl: https://doi.org/10.21203/rs.3.rs-714279/v1

License: (a) This work is licensed under a Creative Commons Attribution 4.0 International License. Read Full License 


\section{Abstract}

Objective: This randomized study aims to evaluate the efficacy and long-term outcome of advanced gastric cancer patients with extensive intraoperative peritoneal lavage (EIPL).

Methods: A total of 150 patients with advanced gastric cancer were enrolled in this study, the patients were randomly allocated to 2 groups: the group of surgery alone (non-EIPL group), the group of surgery plus EIPL (EIPL group). The surviving rate analysis was compared by the Kaplan-Meier method. The prognostic factors were carried out using the Cox appropriate hazard pattern.

Results: Symptom of ileus and abdominal abscess appeared more frequently in the non-EIPL group $(\mathrm{p}<0.05)$. The overall survival (OS) curve and the recurrence free survival (RFS) curve of the EIPL group was better than the non-EIPL group $(p<0.05)$. The EIPL, tumor size, vascular invasion, $N$ stage and $T$ stage were independent prognostic factors of overall survival. The independent risk factors of recurrence free survival (RFS) include EIPL and tumor size. Conclusions: The present study revealed that EIPL can reduce the possibility of perioperative complications including ileus and abdominal abscess. Besides, the overall survival curve and recurrence free survival curve are better in the EIPL group. This technique is easy and not-expensive, therefore EIPL can benefit advanced gastric cancer patients a lot and would be a promising therapeutic strategy in the future.

Trial Registration: Clinical Trials.gov, identifier: NCT02745509, Registered 28 March2016,https://clinicaltrials.gov/ct2/show/NCT02745509?cond=NCT02745509\&draw=2\&rank=1.

\section{Introduction}

Gastric cancer is one of the most common malignant tumors, its morbidity and mortality in China have been increasing in recent years [1,2]. Despite great advances in surgery and other treatment, the 5-year survival rate of gastric cancer is low [3, 4]. One of the main problems after resection is its peritoneal dissemination and recurrence, and peritoneal recurrence is more likely to occur in advanced gastric cancer patients. Although chemotherapy is applied, the prognosis of these patients remains poor [5].

There are some free cancer cells that may still exist after resection. In addition, the surgery itself may lead to the dissemination of tumor cells $[6,7]$, so it is necessary to remove residual tumor cells. Recently, extensive intraoperative peritoneal lavage (EIPL) has got more and more attention, it is a useful treatment which can wash the abdominal cavity completely using $10 \mathrm{~L}$ of physiological saline (up to 10 times). Some researchers found that EIPL plus intraperitoneal chemotherapy can improve the prognosis of patients [8], this technique can eradicate peritoneal free cancer which is beneficial for the recurrence free survival (RFS) of gastric cancer patients $[9,10]$. However, few studies explored the safety and long outcome of EIPL after curative gastrectomy.

In this research, we explore the efficacy and 3-year outcome of advanced gastric cancer patients with technique of EIPL, and then we analyze the possible mechanism. 


\section{Materials And Methods}

\section{Patients}

The study population are advanced gastric cancer patients with clinically $\mathrm{T} 3$ or T4, and M0 disease according to computed tomographic scans and ultrasonographic gastroscopy. The seventh American Joint Committee was used for TNM stage. Each patient signed the informed consent, And this study was approved by the institutional review board of the First Affiliated Hospital of Anhui Medical University, Anqing Municipal Hospital and the First Affiliated Hospital of Wenzhou Medical University.All methods were performed in accordance with the relevant guidelines and regulations.

\section{Inclusion and exclusion criteria}

According to the inclusion and exclusion criteria, patients were included in the research. The inclusion criteria included: 1) All patients were confirmed gastric cancer with T3/4NanyM0; 2) the surgery is definite and complete resection of cancer; 3 ) these patients didn't have heart sickness or any important organ failures; 4) they can keep a long touch with doctors after surgery. The exclusion criteria included: 1) they had previous malignant tumors or various primary tumors; 2 ) they had accepted radiation treatment or chemo treatment previously before the treatment.

\section{Procedure}

If patients were confirmed cT3 or cT4 and M0 disease and suitable for radical gastrectomy, they will be formally included in the study and then randomized. Patients were randomized in the EIPL group or nonEIPL group in a 1:1 ratio. Allocation was performed using sealed opaque envelopes that contained computer-generated random numbers and the procedure to which patients were allocated. Research participants will be randomized to EIPL arm or non-EIPL arm based on random permuted blocks with varying block size of four, assuming equal allocation between treatment arms. After the exploratory operation, the envelopes were opened to determine whether EIPL was applied. Total gastrectomy or partial resection with D2 lymphadenectomy was performed by the guidelines of the Japanese Research Society [11]. For the non-EIPL group, peritoneal lavage was washed using no more than $3 \mathrm{~L}$ of warm saline; In EIPL group, patients received $10 \mathrm{~L}$ or more of saline (1 liter at a time) before closure of the abdomen. Patients were excluded if stage were not detected as T3 or T4 and $\mathrm{M} 0$, in the end 100 patients were finally included in this study between March 2016 and March 2017. Besides, the external population including 50 gastric cancer patients who were hospitalized in the the First Affiliated Hospital of Wenzhou Medical University and Anqing Municipal Hospital from March 2016 to November 2017, and the methods and procedures are consistent with our group.

\section{Data collection and follow-up}

The data of patients' demographic and clinico-pathological data were recorded, including age, gender, tumor location, tumor size, differentiation grade, pathological type and so on. The pathological tumor 
stage was categorized according to the AJCC 7th TNM staging system. The routine laboratory data were listed below: neutrophil, lymphocyte, platelet, CEA and so on.

Peripheral blood tests were obtained within 1 week before surgery and the second days after surgery, the cutoff value of CEA was got according to the normal level. We determine the following indexes (NLR (neutrophil counting/ lymphocyte counting). These two variables were grouped into low group and high group according to the optimal cut-off values which were calculated based on the Youden index [maximum (sensitivity + specificity-1) [12].

The postoperative complications, the length of hospital stay and other outcomes were also recorded in this research, the complications including abscess, leakage, bleeding and so on.

After the operation, the patients received eight 3-week cycles of oral S-1 plus intravenous oxaliplatin. We got their follow-up date through telephones and outpatient visits. This behavior got carried out in normal intervals (each 90 days within three years), until the date of December 2020.

\section{Statistical analysis}

Continuous variables were expressed as mean \pm standard deviation and they were analyzed by Student Ttest; Categorical values were identified by counting (percent) and they were counted by Chi-square test or Fisher exact test. The Kaplan-Meier method was used to compare the prognosis of different groups. The univariate and multivariate analyses were carried out using the Cox proportional hazards model. The whole data explanations got carried out applying SPSS app (17.0 version).

\section{Results}

\subsection{Baseline characteristics}

The baseline characteristics analysis of the 150 patients was shown in Table 1, 109 (72.67\%) were men, and $41(27.33 \%)$ were women. The median age was 67 years (range, $35-80$ years). The basic information in the EIPL group and non-EIPL group had no significant difference. The median follow-up time was 30 months (range $0-45$ months). The 1 - and 3 -year OS rates were $71.0 \%$ and $26.5 \%$, respectively. 
Table 1

The baseline characteristics analysis of the patients.

\begin{tabular}{|c|c|c|c|}
\hline Variable & $\begin{array}{l}\text { Non-EIPL group } \\
(n=75)\end{array}$ & $\begin{array}{l}\text { EIPL group } \\
(n=75)\end{array}$ & $\mathbf{P}$ \\
\hline Age(years) & $66.93 \pm 9.38$ & $64.55 \pm 8.22$ & 0.099 \\
\hline Gender & & & 0.200 \\
\hline male & 51 & 58 & \\
\hline female & 24 & 17 & \\
\hline $\mathrm{BMI}(\mathrm{Kg} / \mathrm{m} 2)$ & $21.51 \pm 2.29$ & $21.64 \pm 3.34$ & 0.786 \\
\hline smoking & & & 0.373 \\
\hline yes & 55 & 50 & \\
\hline no & 20 & 25 & \\
\hline Tumor location & & & 0.260 \\
\hline upper & 21 & 14 & \\
\hline middle & 11 & 17 & \\
\hline low & 43 & 44 & \\
\hline Differentiated grade & & & 0.121 \\
\hline high & 0 & 0 & \\
\hline middle & 55 & 45 & \\
\hline low & 20 & 30 & \\
\hline T stage & & & 0.405 \\
\hline T3 & 4 & 2 & \\
\hline T4 & 71 & 73 & \\
\hline N stage & & & 0.112 \\
\hline NO & 13 & 24 & \\
\hline $\mathrm{N} 1$ & 18 & 12 & \\
\hline N2 & 14 & 17 & \\
\hline N3 & 30 & 22 & \\
\hline Tumor size(cm) & $5.52 \pm 2.21$ & $5.37 \pm 2.32$ & 0.671 \\
\hline Borrmann classification & & & 0.100 \\
\hline
\end{tabular}




\begin{tabular}{|lllll|}
\hline Variable & $\begin{array}{l}\text { Non-EIPL group } \\
(\mathbf{n}=75)\end{array}$ & $\begin{array}{l}\text { EIPL group } \\
(\mathbf{n}=75)\end{array}$ & P \\
\hline II & 7 & 14 & \\
\hline III & 68 & 61 & \\
\hline Neutrophil count $\left(10^{9} / \mathrm{L}\right)$ & $3.49 \pm 1.32$ & $4.71 \pm 8.39$ & 0.215 \\
\hline Lymphocyte count $\left(10^{9} / \mathrm{L}\right)$ & $1.35 \pm 0.47$ & $1.76 \pm 2.48$ & 0.164 \\
\hline NLR & $3.02 \pm 1.96$ & $3.03 \pm 2.20$ & 0.989 \\
\hline Platelet $\left.\left(10^{9} / \mathrm{L}\right)\right)$ & $202.52 \pm 61.39$ & $226.19 \pm 90.94$ & 0.064 \\
\hline
\end{tabular}

\subsection{Surgical outcome after gastrectomy}

Table 2 presented the results of surgery, there were no significant difference in time (surgery to first flatus), postoperative hospital stay, abdominal pain, bleeding, leakage or other blood index between the two groups ( $p>0.05)$, but the symptoms of ileus and abdominal abscess appeared more frequently in the non-EIPL group $(\mathrm{p}<0.05)$. 
Table 2

The outcome after surgery.

\begin{tabular}{|llll|}
\hline Variable & $\begin{array}{l}\text { Non-EIPL group } \\
(\mathbf{n}=75)\end{array}$ & $\begin{array}{l}\text { EIPL group } \\
(\mathbf{n}=75)\end{array}$ & $\mathbf{P}$ \\
\hline Type of surgery & & & 0.242 \\
\hline total & 55 & 61 & \\
\hline distal & 20 & 14 & 0.108 \\
\hline Time(surgery to first flatus, days) & $4.19 \pm 0.99$ & $3.95 \pm 0.87$ & 0.072 \\
\hline Postoperative hospital stay(days) & $15.26 \pm 3.10$ & $14.48 \pm 1.97$ & 0.174 \\
\hline Abdominal pain & $10 / 75$ & $5 / 75$ & 0.003 \\
\hline Ileus & $15 / 75$ & $3 / 75$ & 0.009 \\
\hline Abdominal abscess & $9 / 75$ & $1 / 75$ & 0.246 \\
\hline Leakage & $5 / 75$ & $2 / 75$ & 0.302 \\
\hline Bleeding & $6 / 75$ & $3 / 75$ & 0.552 \\
\hline Neutrophil count $\left(10^{9} / \mathrm{L}\right)$ & $10.36 \pm 3.32$ & $10.03 \pm 3.56$ & 0.817 \\
\hline Lymphocyte cell(109/L) & $1.02 \pm 0.63$ & $1.00 \pm 0.60$ & 0.169 \\
\hline NLR & $13.48 \pm 8.55$ & $11.87 \pm 5.22$ & 0.381 \\
\hline Platelet(10 $/$ L) & $171.00 \pm 59.98$ & $179.73 \pm 60.38$ & \\
\hline
\end{tabular}

\subsection{Overall survival of patients}

Risk factors of overall survival are shown in Table 3. The result showed that the EIPL, Borrmannn classification, tumor size, $\mathrm{N}$ stage, $\mathrm{T}$ stage and vascular invasion were significant indicators. Then multivariate analysis revealed that EIPL, tumor size, vascular invasion, $\mathrm{N}$ stage and T stage were independent prognostic factors (Table 4). The survival curve (Fig. 1) revealed that the prognosis of EIPL group was better than the non-EIPL group $(p<0.001)$, the 3-year survival rate of EIPL group $(38.4 \%)$ was higher than the non-EIPL group (21.7\%). 
Table 3

Univariate analysis of overall survival.

\begin{tabular}{|llll|}
\hline Variable & $\boldsymbol{\beta}$ & $\mathrm{HR}(95 \% \mathrm{Cl}$ for HR) & $\mathbf{P}$ \\
\hline Gender & 0.514 & $1.671(0.983-2.841)$ & 0.058 \\
\hline Age & 0.024 & $1.025(0.994-1.056)$ & 0.114 \\
\hline EIPL/Non-EIPL & -0.991 & $0.371(0.218,0.631)$ & 0.000 \\
\hline Tumor size & 0.192 & $1.211(1.088-1.348)$ & 0.000 \\
\hline Type of Surgery & 0.185 & $1.203(0.653-2.214)$ & 0.553 \\
\hline Tumor location & 0.075 & $0.928(0.689-1.250)$ & 0.622 \\
\hline Borrmann classification & -1.474 & $0.229(0.072-0.731)$ & 0.013 \\
\hline Differentiated grade & 0.491 & $0.612(0.351-1.067)$ & 0.083 \\
\hline T stage & 1.250 & $3.489(1.094-11.130)$ & 0.035 \\
\hline N stage & 0.535 & $1.707(1.339-2.176)$ & 0.000 \\
\hline Vascular invasion & -0.954 & $0.385(0.235-0.632)$ & 0.000 \\
\hline
\end{tabular}

Table 4

Multivariate analysis of overall survival.

\begin{tabular}{|llll|}
\hline Variable & $\boldsymbol{\beta}$ & HR (95\% Cl for HR) & $\mathbf{P}$ \\
\hline EIPL/Non-EIPL & -0.861 & $0.423(0.246-0.727)$ & 0.002 \\
\hline Tumor size & 0.139 & $1.149(1.025-1.289)$ & 0.017 \\
\hline Borrmann classification & -0.268 & $0.765(0.211-2.775)$ & 0.684 \\
\hline T stage & 1.395 & $4.034(1.255-12.971)$ & 0.019 \\
\hline N stage & 0.313 & $1.368(1.034-1.811)$ & 0.029 \\
\hline Vascular invasion & -0.608 & $0.545(0.317-0.935)$ & 0.027 \\
\hline
\end{tabular}

\subsection{Recurrence free survival (RFS) of patients}

The risk factor of RFS included EIPL, N stage, vascular invasion, type of surgery, tumor location, borrmann classification and tumor size (Table 5). EIPL and tumor size were independent risk factors (Table 6). The RFS curve of EIPL group was better than non-EIPL group $(p=0.004)$ (Fig. 2), the recurrence rate of EIPL group (24.7\%) was lower than the non-EIPL group (46.4\%). 
Table 5

Univariate analysis of recurrence free survival.

\begin{tabular}{|llll|}
\hline Variable & $\boldsymbol{\beta}$ & HR $(95 \%$ Cl for HR $)$ & P \\
\hline Gender & 0.465 & $1.592(0.877-2.890)$ & 0.126 \\
\hline Age & 0.013 & $1.013(0.981-1.047)$ & 0.420 \\
\hline EIPL/Non-EIPL & -0.827 & $0.437(0.245-0.781)$ & 0.005 \\
\hline Tumor size & 0.230 & $1.259(1.212-1.414)$ & 0.000 \\
\hline Type of Surgery & 1.081 & $2.948(1.169-7.438)$ & 0.022 \\
\hline Tumor location & 0.422 & $0.656(0.446-0.964)$ & 0.032 \\
\hline Borrmann classification & 1.235 & $0.291(0.090-0.936)$ & 0.038 \\
\hline Differentiated grade & 0.283 & $0.753(0.415-1.367)$ & 0.351 \\
\hline T stage & 1.028 & $2.795(0.869-8.993)$ & 0.085 \\
\hline N stage & 0.499 & $1.647(1.257-2.156)$ & 0.000 \\
\hline Vascular invasion & 0.768 & $0.464(0.265-0.812)$ & 0.007 \\
\hline
\end{tabular}

Table 6

Multivariate analysis of recurrence free survival.

\begin{tabular}{|llll|}
\hline Variable & $\boldsymbol{\beta}$ & HR (95\% Cl for HR) & $\mathbf{P}$ \\
\hline EIPL/Non-EIPL & -0.769 & $0.463(0.254-0.846)$ & 0.012 \\
\hline Tumor size & 0.171 & $1.186(1.039-1.354)$ & 0.012 \\
\hline Type of Surgery & 0.656 & $1.927(0.423-8.777)$ & 0.396 \\
\hline Tumor location & 0.068 & $0.934(0.478-1.823)$ & 0.841 \\
\hline Borrmann classification & 0.062 & $0.940(0.236-3.743)$ & 0.930 \\
\hline N stage & 0.290 & $1.337(0.964-1.854)$ & 0.082 \\
\hline Vascular invasion & 0.364 & $0.695(0.372-1.298)$ & 0.254 \\
\hline
\end{tabular}

\subsection{Patterns of Recurrence}

The recurrence rate of lymph node, node and other organs in the EIPL group and non-EIPL group have no significant difference $(p>0.05)$, but the overall recurrence rate and peritoneum recurrence rate in the EIPL group was lower than the non-EIPL group $(p<0.05)$ (Table 7). 
Table 7

The recurrence location after surgery of advanced gastric cancer patients

\begin{tabular}{|llll|}
\hline Variables & $\begin{array}{l}\text { Surgery alone } \\
(\mathbf{n = 7 5 )}\end{array}$ & $\begin{array}{l}\text { EIPL } \\
(\mathbf{n}=\mathbf{7 5})\end{array}$ & $\mathbf{P}$ \\
\hline Peritoneum & 17 & 7 & 0.026 \\
\hline Lymph node & 5 & 3 & 0.467 \\
\hline liver & 4 & 2 & 0.405 \\
\hline lung & 3 & 1 & 0.311 \\
\hline two place or more & 6 & 4 & 0.513 \\
\hline total & 35 & 17 & 0.002 \\
\hline
\end{tabular}

\section{Discussion}

Peritoneal recurrence are associated with prognosis of gastric cancer $[13,14]$. Previous researches have reported that EIPL combined with intraperitoneal treatment is effective treatment for gastric cancer patients [15], which can reduce the recurrence rate of advanced patients. However, the safety and effect of EIPL alone remain unclear, so this study explore the clinical value of EIPL.

Our results indicated that the overall survival curve and recurrence free survival curve of EIPL group were better than the non-EIPL group, and technique of EIPL was a significant factor of OS and RFS with advanced gastric cancer patients. So EIPL may reduce the recurrence rate of tumor and improve the outcome of patients. Yamamoto K also conducted an RCT of EIPL with pancreatic cancer patients and got the same conclusion [16]. Based on these researches, technique of EIPL need to be applied in abdominal cancers.

The intraoperative bleeding and surgery itself can lead to the residual tumor cell in the abdominal cavities, and this may increase the risk of peritoneal metastasis. In the non-EIPL group, intraperitoneal lavage does not exceed 3 liters of saline, which may be difficult to remove free peritoneal cancer cells. Technique of EIPL can remove free cancer cells and blood in the abdominal cavity by plenty of washing (10 L or more of saline), which can prevent free cancer cells attaching to the peritoneum [17].

In recent years, there have been several reports [18-20] showing that inflammation was linked to poor survival. Inflammation can stimulate the proliferation of malignant tumors cells, promote metastasis and destroy adaptive immunity response [21]. In this study, we found that the preoperative inflammatory index of NLR in the non-EIPL group was lower than the EIPL group. However, the level of postoperative NLR in the non-EIPL group was higher than the EIPL group. As for the patients with high level of NLR, the antitumor immune response of T cells and natural killer cells in the system may be surrounded by a number 
of neutrophils, which may decrease the opportunity to contact with tumor cells [22, 23], so the free peritoneal cancer cells may survive in this course.

This study concluded that the symptoms of ileus appeared more in the non EIPL group than the EIPL group. Besides, EIPL can also reduce the possibility of abdominal abscess, but the complications of bleeding and leakage have no significant difference. Indeed, EIPL is similar to the so-called limiting dilution method [24], this technique can clean up the peritoneal effusion and reduce the risk of infection. The 10 times of regular warm saline can promote intestinal motility and functional recovery, and this may also be helpful for surgeons to find the bleeding place. Besides, the level of NLR in the EIPL group was low after surgery, technique of EIPL can reduce the inflammatory cell and cytokines which play an important role in the development of inflammatory response and tissue damage. So technique of EIPL may be beneficial for the perioperative complications and make patients more comfortable after operation.

Although EIPL could not reduce the recurrence rate of lymph node, node and other organs, the overall recurrence rate and peritoneum recurrence rate in the EIPL group was lower than the non-EIPL group, besides, the overall survival curve and recurrence free survival curve are better in the EIPL group. Currently, only three RCTs are ongoing to explore the long-term efficacy of EIPL of advance gastric cancer. Kuramoto et al[8] concluded that the peritoneal recurrence rate of the EIPL group was significantly lower than that of the non-EIPL $(6.7 \%$ vs. $45.8 \%, P=0.013)$, there is no difference in recurrence rate for liver transfer, lymph node, and other organ transfer cases between the two groups, which is similar to our study. Another advantage is that IPC is not taken in our study, it may remove side effects associated with chemotherapy and confound the effect of EIPL. Misawa K [25] conducted an RCT indicating that peritoneal recurrence-free survival was not significantly different between the EIPL group and non-EIPL group. The 3-year overall survival rate and RFS rate was better than our study, and the reason is that the proportion of T4 (49.5\%) and N3 (28.1\%) is smaller than our study population (T4:96.0\%, N3:34.7\%). The value of EIPL may be related to the stage of $\mathrm{T}$ status and $\mathrm{N}$ status. The patients of our study (more cases of T4 and N3) have higher risk of recurrence, and the reduction of recurrence rate is significant in the EIPL group. One RCT is still ongoing based in Singapore [26], eligible patients having cT3 or cT4 with M0 disease are also in their criteria, but our study collected more clinical information and explored the safety and efficacy of EIPL group. Our study showed that technique of EIPL can reduce the perioperative complications of patients.

Our study has several limitations. First, we analyze only advanced gastric cancer patients, they may not on behalf of all patients. Second, more cases need to verify our results.

In conclusion, EIPL can reduce the possibility of perioperative complications including ileus and abdominal abscess. Besides, the overall survival curve and recurrence free survival curve are better in the EIPL group. This technique is easy and not-expensive. Therefore, EIPL can benefit advanced gastric cancer patients a lot and would be a promising therapeutic strategy in the future. 


\section{Abbreviations}

EIPL

extensive intraoperative peritoneal lavage

NLR

neutrophils to lymphocytes ratio

PLR

platelets to lymphocytes ratio

OS

overall survival

RFS

recurrence free survival

\section{Declarations}

\section{Acknowledgements}

None.

\section{Funding}

None.

\section{Availability of data and materials}

The datasets used and/or analyzed during the current study are available from the corresponding author on reasonable request.

\section{Author contributions}

Li-xiang Zhang and Chuan-hong Li wrote the main manuscript text, Jun Ma, Lei Chen and Zhi-jian Wei prepared figures and tables. A-Man Xu, Wen-xiu Han conceived and designed the study and assisted in the drafting of the manuscript. All authors have read and approved the final manuscript.

\section{Corresponding author}

Correspondence to Wen-xiu Han.

\section{Ethics approval and consent to participate}

this study was approved by the institutional review board of First Affiliated Hospital of Anhui Medical University, Anqing Municipal Hospital and First Affiliated Hospital of Wenzhou Medical University.All methods were performed in accordance with the relevant guidelines and regulations . 
Consent for publication $₫$ Not applicable.

Competing interests

The authors have no conflict of interest to disclose.

\section{Author information}

Affiliations

Department of general Surgery, First Affiliated Hospital of Anhui Medical University, Hefei, Anhui 230022, China

Li-xiang Zhang, Chuan-hong Li, A-Man Xu, Wen-xiu han, Zhi-jian Wei

Department of Surgical Oncology, Anqing Municipal Hospital, Anqing, Anhui 246004, China

Jun Ma

Department of General Surgery, First Affiliated Hospital of Wenzhou Medical University, Wenzhou Zhejiang 325035, China

Lei Chen

\section{References}

1. Naghavi M. The global burden of cancer. JAMA Oncol. 2015;1:505-27.

2. Liu Y, Zhang Q, Ren C, Ding Y, Jin G, et al. A germline variant N375S in MET and gastric cancer susceptibility in a Chinese population. J Biomed Res. 2012;26:315-8.

3. Wang W, Li Y-F, Sun X-W, et al. Prognosis of 980 patients with gastric cancer after surgical resection [J]. Chinese Journal of Cancer. 2010;29(11):923-30.

4. lixiang zhang, zhijain wei, aman xu. A simple model established by blood markers predicting overall survival after radical resection of type II and III Adenocarcinomas of the Esophagogastric Junction, a preprint at https://www.researchsquare.com/article/rs-11713/v1.

5. Spratt JS, Adcock RA, Muskovin M, SherrillW, McKeown J. Clinical delivery system for intraperitoneal hyperthermic chemotherapy. Cancer Res. 1980;40(2):256-60.

6. Yamamoto K, Shimada S, Hirota M,et al. EIPL (extensive intraoperative peritoneal lavage) therapy significantly reduces peritoneal recurrence after pancreatectomy in patients with pancreatic cancer.Int. J Oncol. 2005;27:1321-8.

7. Shimada S, Tanaka E, Marutsuka T. et ah Extensive intraoperative peritoneal lavage and chemotherapy for gastric cancer patients with peritoneal free cancer cells. Gastric Cancer 5:168172,2002 . 
8. Kuramoto M, Shimada S, Ikeshima S, Matsuo A, Yagi Y, Matsuda M, et al. Extensive intraoperative peritoneal lavage as a standard prophylactic strategy for peritoneal recurrence in patients with gastric carcinoma. Ann Surg. 2009;250:242-6.

9. Shimada S, Tanaka E, Marutsuka T. et ah Extensive intraoperative.

10. peritoneal lavage. and chemotherapy for gastric cancer patients with peritoneal free cancer cells. Gastric Cancer. 2002;5:168-72.

11. Misawa K, Mochizuki Y, Sakai M,et al. Randomized clinical trial of extensive intraoperative peritoneal lavage versus standard treatment for resectable advanced gastric cancer (CCOG 1102 trial). Br J Surg. 2019;106:1602 1610.

12. Japanese Gastric Cancer Association. Japanese gastric cancer treatment guidelines 2010 (ver. 3). Gastric Cancer. 2011;14(2):113-23.

13. Youden WJ. Index for rating diagnostic tests. Cancer. 1950;3:32-5.

14. Ulysses R Jr, Adiana VS, Bruno Z, et al. Does the intraoperative peritoneal.

15. lavage cytology add. prognostic information in patients with potentially curative gastric resection? J Gastrointest Surg. 2006;10:170 - 177.

16. Nakagawa A, Nashimoto A, Yabusaki H. Role of staging laparoscopy with peritoneal lavage cytology of locally advanced gastric cancer. Gastric Cancer. 2007;10:29-34.

17. Kuramoto M, Shimada S, Ikeshima S, et al. Extensive intraoperative peritoneal lavage as a standard prophylactic strategy for peritoneal recurrence in patients with gastric carcinoma. Ann Surg. 2009;250(2):242-6.

18. Yamamoto K, Shimada S, Hirota M, et al. EIPL(extensive intraoperative peritoneal lavage) therapy significantly reduces peritoneal recurrence after pancreatectomy in patients with pancreatic cancer. Int J Oncol. 2005;27:1321-8.

19. Arita T, Ichikawa D, Konishi H, Komatsu S, Shiozaki A, Hiramoto H, et al. Increase in peritoneal recurrence induced by intraoperative hemorrhage in gastrectomy. Ann Surg Oncol. 2015;22:758-64.

20. Grivennikov SI, Greten FR, Karin M. Immunity, inflammation, and cancer. Cell. 2010;140:883-99.

21. Szkandera J, Absenger G, Liegl-Atzwanger B, Pichler M, Stotz M, Samonigg $H$,et al. Elevated preoperative neutrophil/lymphocyte ratio is associated with poor prognosis in soft-tissue sarcoma patients. British journal of cancer. 2013;108:1677-83.

22. Que Y, Qiu H, Li Y, Chen Y, Xiao W, Zhou Z, et al. Preoperative platelet-lymphocyte ratio is superior to neutrophil-lymphocyte ratio as a prognostic factor for soft-tissue sarcoma. BMC Cancer. 2015;15:648.

23. Mantovani A, Allavena P, Sica A, Balkwill F. Cancer-related inflammation. Nature. 2008;454:436-44.

24. Grivennikov SI, Greten FR, Karin M. Immunity, inflammation, and cancer, Cell140 (2010) 883-899.

25. Smyth MJ, Dunn GP, Schreiber RD. Cancer immunosurveillance and immunoediting: the roles of immunity in suppressing tumor development and shaping tumor immunogenicity [J]. Adv Immunol. 2006;90:1-50. 
26. Ruiz-Tovar J, Llavero C, Muz JL, Zubiaga L, Diez M. Effect of peritoneal lavage with clindamycingentamicin solution on post-operative pain and analytic acute-phase reactants after laparoscopic sleeve gastrectomy. Surg Infect (Larchmt). 2016;17(3):357-62. doi:10.1089/sur.2015.196.

27. Misawa K, Mochizuki Y, Ohashi N, et al. A randomized phase III trial exploring the prognostic value of extensive intraoperative peritoneal lavage in addition to standard treatment for resectable advanced gastric cancer: CCOG 1102 study. Jpn J Clin Oncol. 2014;44:101-3.

28. Kim G, Chen E, Tay AY, et al. Extensive peritoneal lavage after curative gastrectomy for gastric cancer (EXPEL): study protocol of an international multicentre randomised controlled trial. Jpn J Clin Oncol. 2017;47(2):179-84.

\section{Figures}

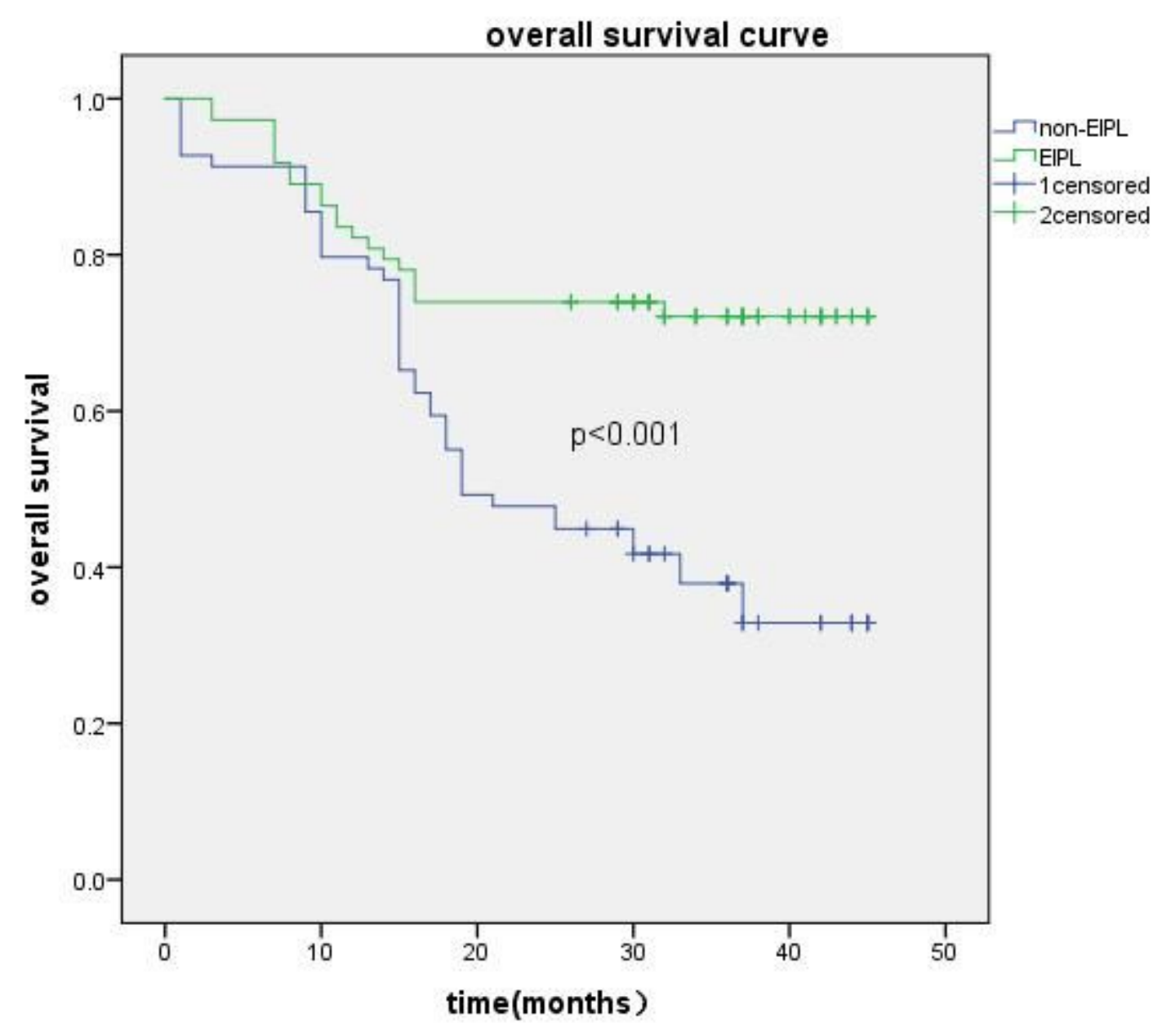

\section{Figure 1}

The Kaplan-Meier curve of overall survival in the non- EIPL group and EIPL group 


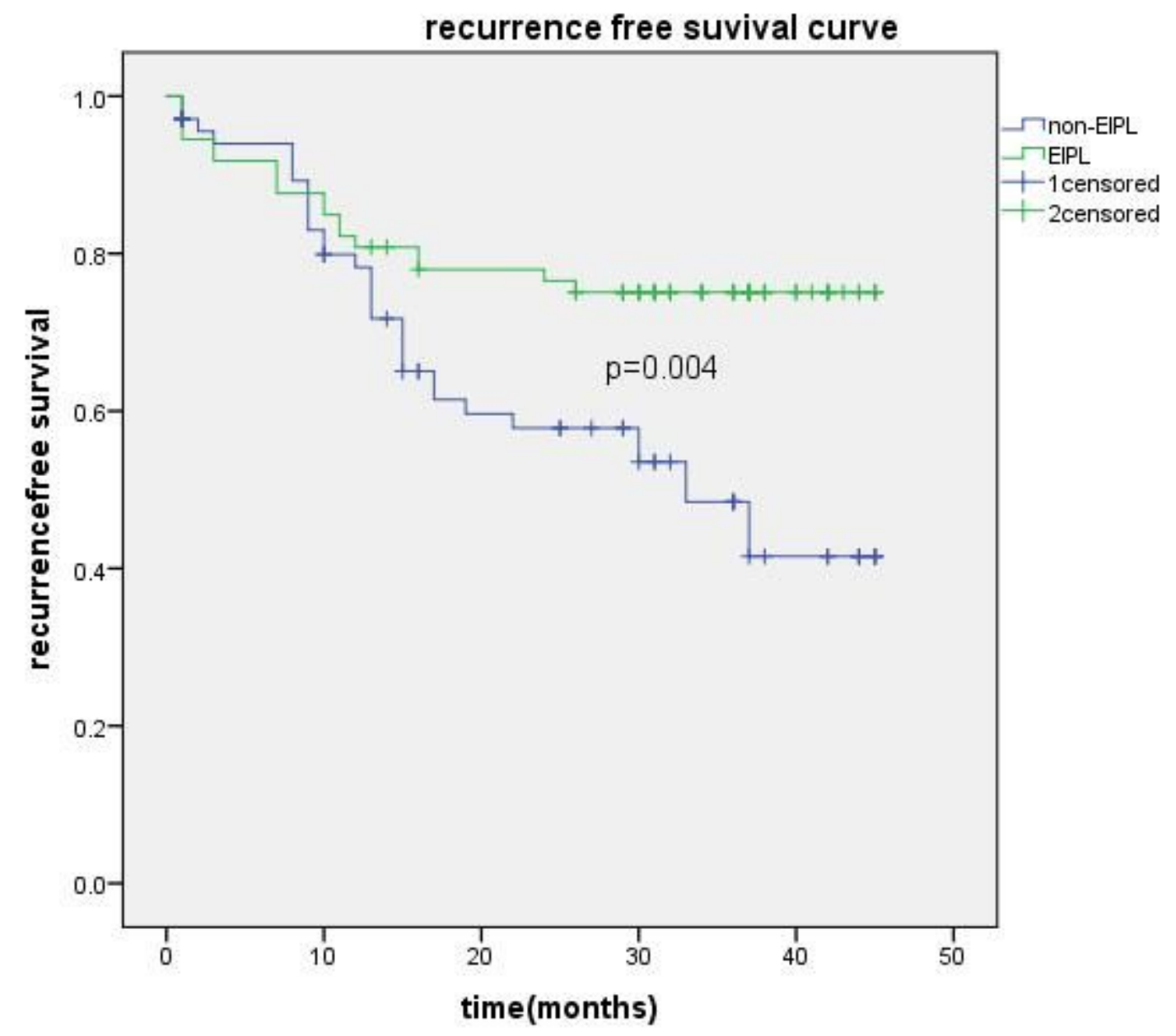

Figure 2

The Kaplan-Meier curve of recurrence free survival in the non-EIPL group and EIPL group 